\title{
Antidepressant effects of Kai-Xin-San in fluoxetine-resistant depression rats
}

\author{
X.Z. Dong ${ }^{1 *}$, D.X. Wang ${ }^{1 *}$, Y.P. $\mathrm{Lu}^{1,2 *}$, S. Yuan ${ }^{1}$, P. $\mathrm{Liu}^{1}$ and Y. $\mathrm{Hu}^{1}$
}

${ }^{1}$ Department of Clinical Pharmacology, General Hospital of Chinese People's Liberation Army, Beijing, China

${ }^{2}$ Department of Chinese Medicine, Shanxi University of Traditional Chinese Medicine, Jinzhong, Shanxi, China

\begin{abstract}
This study aimed to investigate the antidepressant effect and the mechanism of action of Kai-Xin-San (KXS) in fluoxetineresistant depressive (FRD) rats. Two hundred male Wistar rats weighing $200 \pm 10 \mathrm{~g}$ were exposed to chronic and unpredictable mild stresses (CUMS) for 4 weeks and given fluoxetine treatment simultaneously. The rats that did not show significant improvement in behavioral indexes were chosen as the FRD model rats. These rats were randomly divided into four groups: FRD model control; oral fluoxetine and aspirin; oral KXS at a dose of $338 \mathrm{mg} \cdot \mathrm{kg}^{-1} \cdot$ day $^{-1}$; and oral KXS at a dose of $676 \mathrm{mg} \cdot \mathrm{kg}^{-1} \cdot \mathrm{day}^{-1}$. Rats continued to be exposed to CUMS and underwent treatment once a day for 3 weeks, then cytokine (COX-2, IFN- $\gamma$, IL-1 $\beta$, IL-2, IL-4, IL-6, IL-10, TGF- $\beta$, and TNF- $\alpha$ ) levels in the hippocampus and serum, and organ coefficients were measured. Both doses of KXS improved the crossing and rearing frequencies, sucrose-preference index, and body weight in FRD rats. $\mathrm{KXS}$ at a dose of $338 \mathrm{mg} \cdot \mathrm{kg}^{-1}$. day ${ }^{-1}$ reduced COX-2, IL-2, IL-6, TNF- $\alpha$ levels, increased IL-10 level in the hippocampus, and reduced IL-2 and TNF- $\alpha$ levels in serum. KXS at a dose of $676 \mathrm{mg} \cdot \mathrm{kg}^{-1} \cdot$ day $^{-1}$ reduced TNF- $\alpha$ level in the hippocampus, reduced IL-2 and TNF- $\alpha$ levels in serum, and increased IFN- $\gamma$ and IL-10 levels in the hippocampus and serum. There were no significant differences in organ-coefficients of the spleen among and between groups. The results suggested that oral administration of KXS in FRD rats was effective in improving behavior disorders by influencing various inflammatory pathways.
\end{abstract}

Key words: Kai-Xin-San; Fluoxetine-resistant depression; Chronic unpredictable mild stress; Inflammatory factor; Anti-depression

\section{Introduction}

Major depression is a common and sometimes fatal disorder that has a worldwide prevalence greater than $15 \%$. It is estimated that major depressive disorder will be the second largest contributor to the global burden of disease by 2020 . Despite considerable advances in the treatment of major depressive disorder in the past few years, treatment-resistant depression (TRD) remains a common condition that affects approximately $30 \%$ of this population (1). Therefore, identifying a potential drug that is effective in treating resistant depression with low toxicity is important.

Many studies have shown that increased plasma concentrations of interleukin (IL)-1, IL-6, and tumor necrosis factor (TNF)- $\alpha$ were found in major depression patients with a history of poorer response to antidepressants than in treatment-responsive patients (2-4). Similarly, patients with increased inflammatory activity before treatment have been reported to be less responsive to antidepressants
(5-7). Given these findings, researchers hypothesized that inflammation may influence the effects of antidepressants. To support this hypothesis, the chronic unpredictable mild stress (CUMS) paradigm was tested by administering lipopolysaccharide (LPS) daily before the stressor. It was found that pretreatment with LPS, mimicking inflammation, had no significant effect on depressionrelated behaviors but attenuated the antidepressant action of fluoxetine significantly, suggesting that inflammation might play a role in the pathophysiology of antidepressant resistance (8).

Kai-Xin-San (KXS) (9) is a well-known formula that was first recorded in an ancient Chinese book: "Tai Ping Hui Min He Ji Ju Fang". KXS consists of Ginseng (Panax ginseng C.A. Meyer), hoelen (Wolf Poria cocos, Schw), polygala (Polygala tenuifolia Willd), and Acorus (Acorus tatarinowii Schott) in a ratio of 3:3:2:2. For thousands of years, it has been a renowned Chinese herbal formula for

Correspondence: P. Liu: <cpi301@163.com> | Y. Hu: <huyuan1980619@126.com>

${ }^{*}$ These authors contributed equally to this study.

Received December 31, 2016 | Accepted June 27, 2017 
treating depression and ameliorating various learning and memory deficits, such as desolation, moodiness, and forgetfulness.

Our previous studies in mice models have indicated that KXS has antidepressant-like effects as demonstrated by the tail suspension test and forced swim test. It also significantly elevated the levels of central monoamine neurotransmitters, including 5-hydroxytryptamine, dopamine, and noradrenaline $(10,11)$. Simultaneously, KXS could ameliorate chronic fatigue syndrome by promoting proliferation of splenocytes in mice and modulate the detrimental effects of cytokines (12). KXS exerts its antidepressant-like and nootropic effect in a CUMS model by modulating the hypothalamic-pituitary-adrenal axis, monoamine neurotransmitter levels, and cholinergic systems (13).

Based on the biological effects of KXS that have been explored previously, the current study aimed to assess its potential antidepressant action and its influence on inflammatory processes to identify potential mechanisms in fluoxetine-resistant depressive rats.

\section{Material and Methods}

\section{Reagents and drugs}

Fluoxetine was purchased from Eli Lilly and Company (USA). Aspirin was purchased from Bayer Medicines Company (Germany). ELISA kits for TNF- $\alpha$, TGF- $\beta$, IFN- $\gamma$, COX-2, IL-1 $\beta$, IL-2, IL-4, IL-6, and IL-10 were purchased from R\&D Company (USA). KXS was purchased from the LvYe Medicinal Material Company (China). KXS was supplied in powder form, which was derived from a mixture of the aqueous extract as described previously (14).

\section{Animals}

In total, 200 male Wistar rats weighing $200 \pm 10 \mathrm{~g}$ were obtained from the Animal Breeding Center of the PLA General Hospital (Beijing, China). All rats were kept in a temperature- $\left(23 \pm 2^{\circ} \mathrm{C}\right)$ and humidity-controlled $(60 \pm 10 \%)$ facility on a 12-h light/dark cycle with free access to food and water. All animal experimental protocols were approved by the Animal Experimentation Ethics Committee of General Hospital of Chinese PLA. All animal handling procedures were performed in compliance with the 'Principles of Laboratory Animal Care' and the Chinese legislation for the use and care of laboratory animals.

\section{Fluoxetine-resistant depression model and drug administration}

Twelve rats were randomly assigned as the normal control group, housed undisturbed in 4 per cage without contact with the stressed animals. The remaining 188 rats were used to replicate the CUMS model following the established protocol (15). Rats received 4 weeks of stress stimulations, which consisted of high-speed agitation (10 min), immobilization (2 h), tilted cage (12 h), deprivation of food or water (24 h), continuous illumination (24 h), and forced swimming in ice water (5 min). Rats were randomly assigned one stimulation daily from 3:00-5:00 pm over 4 weeks, and the stressed rats were housed in individual cages to sustain the depressive state until the end of the experiment. Among the 188 rats, 12 were randomly assigned as depression model control (CUMS group), treated with CUMS stimulations only (administered water orally, with no fluoxetine), used for screening the FRD rats; the other 176 rats were administered fluoxetine (20 $\mathrm{mg} \cdot \mathrm{kg}^{-1} \cdot$ day $^{-1}$, orally) for 4 weeks simultaneously. After 4 weeks, behavior tests (crossing frequency, rearing times, and sucrose preference) were performed. Among the 176 rats, the rats whose behavior index had no significant improvement compared with the CUMS rats and was significantly lower than that of normal rats were chosen as the FRD model. Ultimately, 48 rats met the criteria and were randomly divided into four groups. The rats were sequentially given CUMS stress and treated with water ( $\mathrm{n}=12$, FRD model group), fluoxetine $\left(20 \mathrm{mg} \cdot \mathrm{kg}^{-1} \cdot \mathrm{day}^{-1}\right)$ combined with aspirin $\left(20 \mathrm{mg} \cdot \mathrm{kg}^{-1} \cdot \mathrm{day}^{-1}\right)(\mathrm{n}=12$, Flu + Aspirin group), $\mathrm{KXS}$ at $338 \mathrm{mg} \cdot \mathrm{kg}^{-1} \cdot \mathrm{day}^{-1}(\mathrm{n}=12, \mathrm{KXS}-$ 338 group), and $\mathrm{KXS}$ at $676 \mathrm{mg}^{-1} \cdot \mathrm{kg}^{-1} \cdot \mathrm{day}^{-1}(\mathrm{n}=12$, KXS-676 group) orally at 9:00-10:00 am for 3 weeks. Twelve rats in the normal control group were continuously administered water. The time interval between fluoxetine and aspirin in the Flu + Aspirin group was $30 \mathrm{~min}$.

\section{Open-field test}

The locomotor activity was assessed to detect immobility or changes in motor activity in the open-field test performed on days 0,28 , and 46 of the experiment. The open-field apparatus was a cubic open field arena measuring $80 \mathrm{~cm}$ in length, $80 \mathrm{~cm}$ in width, and $60 \mathrm{~cm}$ in height. The box floor was divided into 25 squares ( 5 squares long $\times 5$ squares wide). Rats were placed individually into the center of the arena and allowed to explore freely for $5 \mathrm{~min}$. The floor was wiped cleaned with $70 \%$ ethanol between tests. The number of square line crossings with all four paws and rearing (when the rat stood on its hind limbs) were recorded.

\section{Sucrose-preference test}

The tests were performed on days 0,28 , and 46 of the experiment. Before the sucrose-preference test, rats were deprived of food and water for $24 \mathrm{~h}$ and then fed with two pre-weighted bottles containing $1 \%$ sucrose solution and water for $1 \mathrm{~h}$. Intake was measured by weighing the bottles before and after each test. All tests were carried out in the home cage to minimize extraneous novelty and disturbance. The sucrose preference was calculated as sucrose intake/total water intake (sucrose intake + water intake). Anhedonia was defined as a reduction in sucrose preference relative to baseline levels.

\section{Enzyme-linked immunosorbent assay}

After the experiment, rats were anesthetized with an intraperitoneal injection of $10 \%$ chloral hydrate $(0.35 \mathrm{~mL} / 100 \mathrm{~g}$ 
body weight). Blood samples were collected, and serum was separated from aliquots of blood samples to determine the levels of serum inflammatory cytokines. The whole brain was then quickly removed with scissors, and the hippocampus was isolated, frozen in liquid nitrogen, and stored at $-80^{\circ} \mathrm{C}$ for further biochemical analysis. The levels of cytokines (COX-2, IFN- $\gamma, \mathrm{IL}-1 \beta$, IL-2, IL-4, IL-6, IL-10, TGF- $\beta$, and TNF- $\alpha$ ) in the hippocampus and serum were measured using a paired antibody quantitative ELISA kit according to the manufacturer's instructions. The plates were measured using a microtiter plate reader (Perkin-Elmer, USA). Data are reported as $\mathrm{ng} / \mathrm{mL}$.

\section{Statistical analysis}

Data are reported as means \pm SE. Differences between groups were analyzed by one-way ANOVA followed by Dunnett's test. Data were analyzed statistically using SPSS 17.0 software (USA). $P$ values less than 0.05 were considered statistically significant.

\section{Results}

\section{Fluoxetine-resistant depression model and behavior evaluation}

Results of the open-field tests and sucrose-preference tests showed that before CUMS stress treatment (day 0), the number of line crossings and rearings, sucrosepreference index and body weights between groups had no significant differences. After 4 weeks, compared with normal control, the model rats, which were treated with
CUMS stress and fluoxetine (day 28), showed a significant decrease in crossing frequency, rearing frequency, sucrose-preference index, and body weight $(P<0.05)$. After 3 weeks of treatment (day 46), compared with the model control (treated with water), rats treated with CUMS stress, and fluoxetine + aspirin showed a significant increase in crossing frequency and sucrose-preference index. Both doses of KXS reverted the reduced crossing frequency, rearing frequency, sucrose-preference index and body weights in fluoxetine-resistant rats $(p<0.05)$ (Table 1).

\section{Cytokines in the hippocampus}

ELISA experiments showed that levels of COX-2, IL-2, and TNF- $\alpha$ were increased significantly and levels of IL-10 were reduced in the hippocampus of fluoxetine-resistant rats, compared with normal rats $(P<0.05)$. Fluoxetine in combination with aspirin decreased the levels of COX-2, IL-2, IL-4, and TNF- $\alpha$ in the hippocampus $(p<0.05)$. KXS at a dose of $338 \mathrm{mg} \cdot \mathrm{kg}^{-1} \cdot$ day $^{-1}$ reduced levels of COX-2, IL-2, IL-6 and TNF- $\alpha$, and increased IL-10 levels $(P<0.05)$. $\mathrm{KXS}$ at a dose of $676 \mathrm{mg} \cdot \mathrm{kg}^{-1}$. day $^{-1}$ lowered TNF- $\alpha$ levels and increased IFN- $\gamma$ levels in the hippocampus $(p<0.05)$ (Figure 1).

\section{Cytokines in serum}

Compared with normal rats, levels of IL-2 and TNF- $\alpha$ were increased significantly, and the levels of IL-10 were reduced in the model group (Figure 2). This is similar to the results found in the hippocampus. Fluoxetine in

Table 1. Effects of Kai-Xin-San (KXS) on behaviors measured on days 0,28 and 46, in fluoxetine-resistant depression (FRD) rats exposed to chronic and unpredictable mild stress model.

\begin{tabular}{lccccc}
\hline Index/Days & Normal & Model & Flu + Aspirin & KXS-338 & KXS-676 \\
\hline Crossings & & & & & \\
D0 & $175.9 \pm 0.2$ & $180.6 \pm 52.3$ & $169.0 \pm 14.5$ & $167.1 \pm 45.8$ & $163.9 \pm 25.4$ \\
D28 & $160.9 \pm 20.1$ & $107.3 \pm 27.9^{\# \#}$ & $111.8 \pm 29.8^{\# \#}$ & $106.5 \pm 22.2^{\# \#}$ & $119.1 \pm 24.8^{\# \#}$ \\
D46 & $152.5 \pm 38.6$ & $101.5 \pm 19.4^{\# \#}$ & $128.1 \pm 28.6^{*}$ & $122.1 \pm 15.9^{*}$ & $135.0 \pm 28.2^{*}$ \\
Rearings & & & & & \\
D0 & $29.1 \pm 9.7$ & $26.8 \pm 7.4$ & $25.8 \pm 6.2$ & $24.4 \pm 5.5$ & $22.0 \pm 4.6$ \\
D28 & $20.1 \pm 5.9$ & $12.0 \pm 4.9^{\# \#}$ & $11.4 \pm 5.9^{\# \#}$ & $13.9 \pm 4.2^{\# \#}$ & $15.0 \pm 2.6^{\# \#}$ \\
D46 & $14.5 \pm 5.0$ & $8.9 \pm 3.5^{\# \#}$ & $8.4 \pm 4.1$ & $9.3 \pm 4.2$ & $9.8 \pm 5.5^{*}$ \\
Sucrose preference $(\mathrm{mL})$ & & & & & \\
D0 & $86.2 \pm 17.3$ & $89.6 \pm 15.2$ & $83.2 \pm 16.9$ & $81.9 \pm 10.5$ & $86.4 \pm 12.4$ \\
D28 & $97.2 \pm 6.7$ & $79.5 \pm 3.2^{\# \#}$ & $75.6 \pm 7.9^{\# \#}$ & $73.4 \pm 14.6^{\# \#}$ & $78.8 \pm 6.6^{\# \#}$ \\
D46 & $98.2 \pm 5.4$ & $72.2 \pm 13.1^{\#}$ & $86.2 \pm 9.1^{*}$ & $87.5 \pm 11.1^{*}$ & $92.3 \pm 4.1^{* *}$ \\
Body weight (g) & & & & & \\
D0 & $250.1 \pm 8.7$ & $241.2 \pm 7.4$ & $235.3 \pm 10.4$ & $235.4 \pm 11.2$ & $242.5 \pm 11.9$ \\
D28 & $416.2 \pm 23.3$ & $335.1 \pm 7.5^{\# \#}$ & $339.9 \pm 12.1^{\# \#}$ & $335.3 \pm 16.2^{\# \#}$ & $331.5 \pm 16.3^{\# \#}$ \\
D46 & $446.5 \pm 22.7$ & $351.9 \pm 11.1^{\# \#}$ & $349.4 \pm 10.6$ & $371.4 \pm 18.6^{*}$ & $390.4 \pm 22.4^{* *}$ \\
\hline
\end{tabular}

Data are reported as means $\pm S D, n=12$. Groups: FRD model control (Model), oral fluoxetine and aspirin (Flu + Aspirin), oral KXS at a dose of $338 \mathrm{mg} / \mathrm{kg}$, and $676 \mathrm{mg} / \mathrm{kg} .{ }^{\#} \mathrm{P}<0.05,{ }^{\#} \mathrm{P}<0.01$ compared to untreated Normal group. ${ }^{*} \mathrm{P}<0.05$, ${ }^{* \star} \mathrm{P}<0.01$ compared to Model control group. 

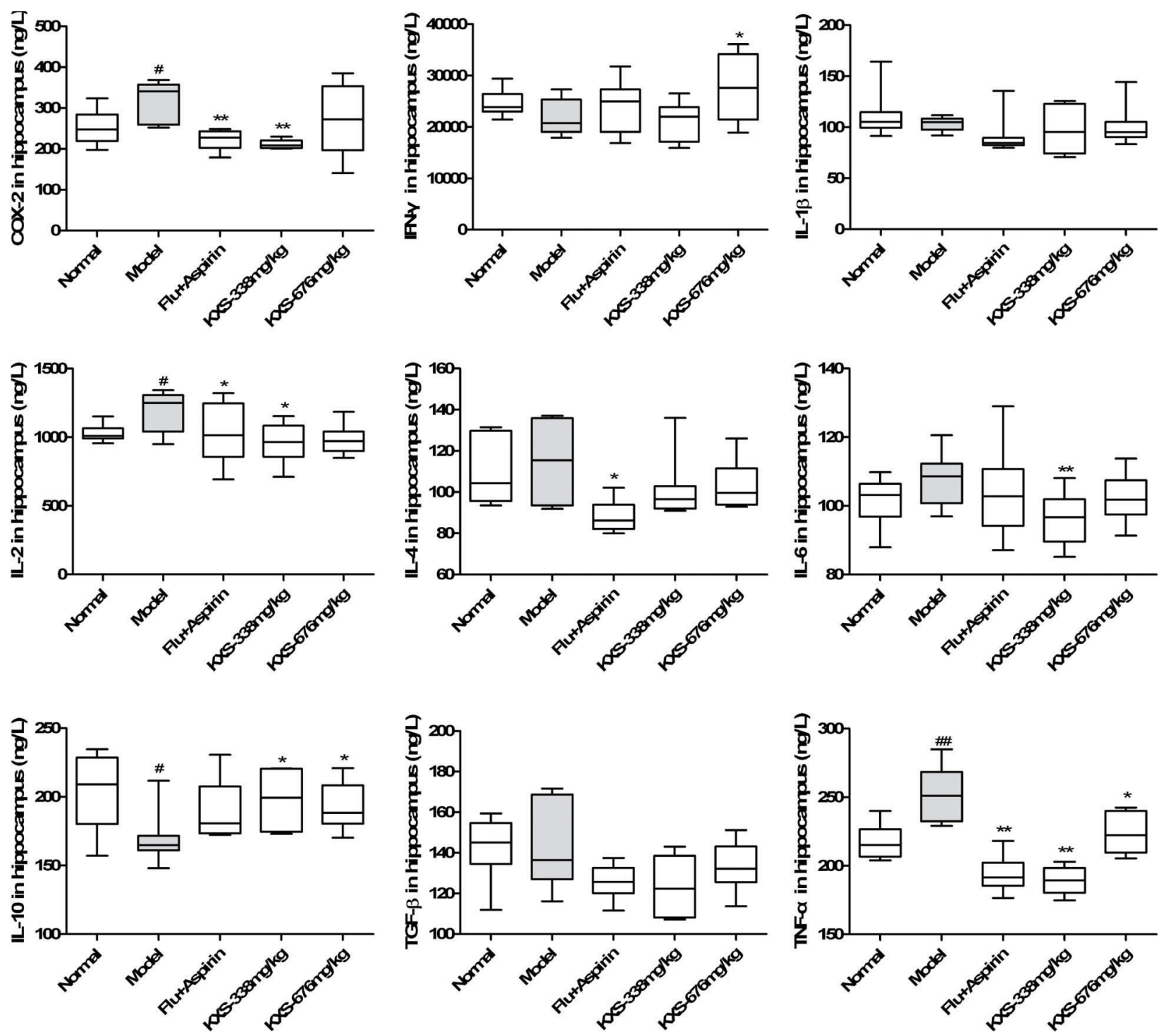

Figure 1. Effects of Kai-Xin-San (KXS) on cytokines in the hippocampus in fluoxetine-resistant depression (FRD) rats exposed to chronic and unpredictable mild stress model and randomly divided into four groups: FRD model control (Model), oral fluoxetine and aspirin (Flu + Aspirin), oral KXS at a dose of $338 \mathrm{mg} / \mathrm{kg}$, and $676 \mathrm{mg} / \mathrm{kg}$. Data are reported as means $\pm S D, n=12$. ${ }^{\#} P<0.05$, ${ }^{\# \#} P<0.01$ compared to untreated Normal group. ${ }^{*} \mathrm{P}<0.05$, ${ }^{\star *} \mathrm{P}<0.01$ compared to Model control group (ANOVA followed by Dunnett's test).

combination with aspirin decreased TNF- $\alpha$ levels and increase IL-10 levels in serum. KXS at both doses reduced levels of $\mathrm{IL}-2$ and $\mathrm{TNF}-\alpha, \mathrm{KXS}$ at a dose of $676 \mathrm{mg} \cdot \mathrm{kg}^{-1} \cdot$ day $^{-1}$ also increased IFN- $\gamma$ and IL-10 levels in serum $(\mathrm{P}<0.05)$.

\section{Spleen coefficient}

Compared with that of normal rats, the spleen coefficient in fluoxetine-resistant rats was increased significantly $(P<0.05)$, but treatment with fluoxetine in combination with aspirin or with KXS (both doses) had no significant influence on the spleen coefficient (Figure 3).

\section{Discussion}

Depression is a psychological illness with high levels of disability and mortality, and is one of the most prevalent diseases in the world. In recent years, numerous studies have demonstrated a clear relationship between inflammation and the development of depression. For example, levels of IL-2, IL-6 in peripheral blood of patients with depression were significantly increased (16), levels of IL-2, IL-6, TNF- $\alpha$ in patients with first-episode depression were greater than that in normal patients (17), and overexpression of 

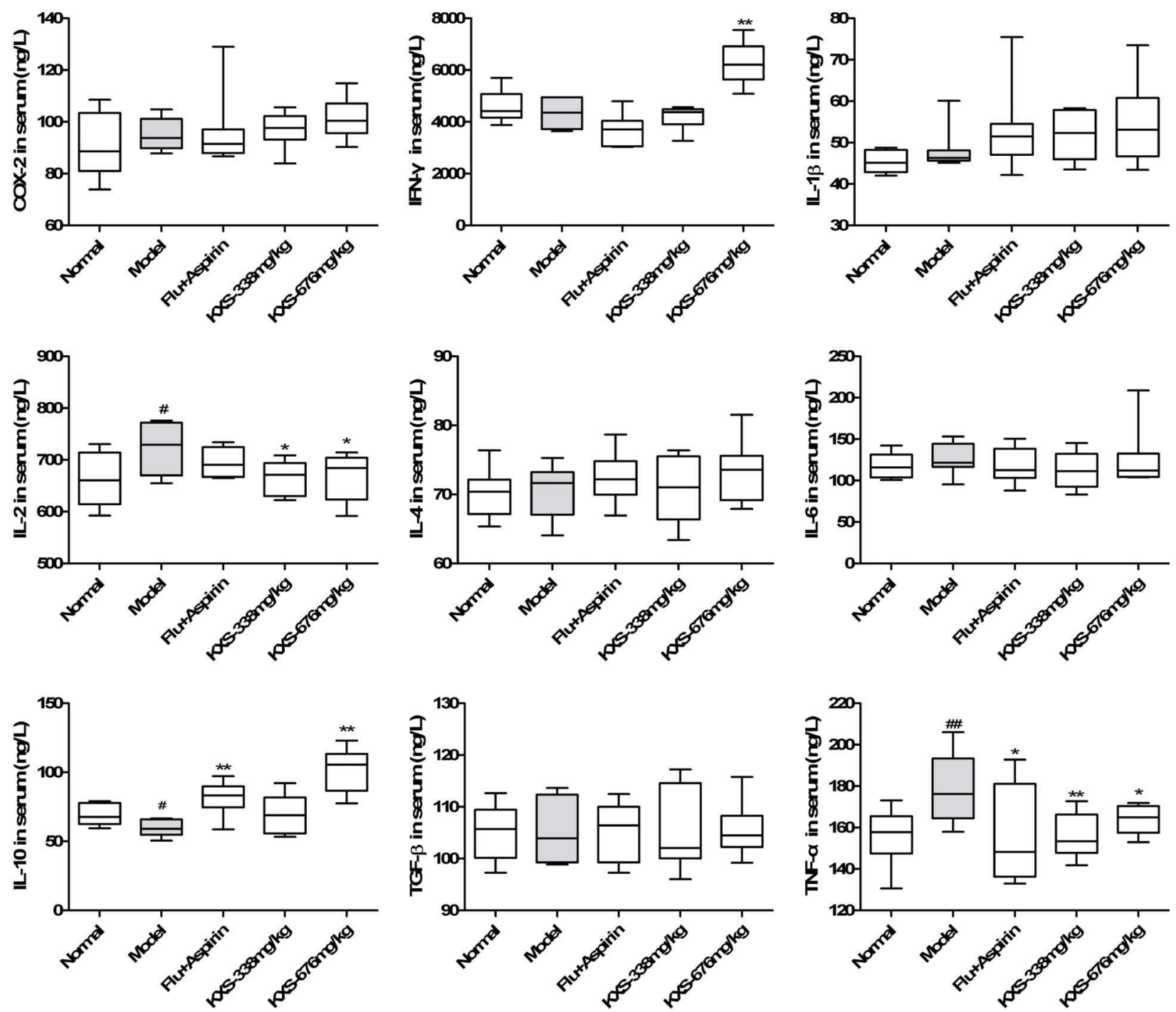

Figure 2. Effects of Kai-Xin-San (KXS) on cytokines in serum in fluoxetine-resistant depression (FRD) rats exposed to chronic and unpredictable mild stress model and randomly divided into four groups: FRD model control (Model), oral fluoxetine and aspirin (Flu + Aspirin), oral KXS at a dose of $338 \mathrm{mg} / \mathrm{kg}$, and $676 \mathrm{mg} / \mathrm{kg}$. Data are reported as means $\pm \mathrm{SD}, \mathrm{n}=12 .{ }^{\#} \mathrm{P}<0.05,{ }^{\# \#} \mathrm{P}<0.01 \mathrm{compared}$ to untreated Normal group. ${ }^{*} \mathrm{P}<0.05,{ }^{* \star} \mathrm{P}<0.01$ compared to Model group.

COX was found in the hippocampus of depressive rat models (18-20). These substances can inhibit the development of nerve cells, activate the hypothalamicpituitary-adrenal axis simultaneously, increase the secretion of glucocorticoids and promote apoptosis (21). These findings suggest that changes in serum cytokine (such as COX, IL-2 and TNF- $\alpha$ ) concentrations play an important role in the development and pathophysiology of depressive disorders. Depression is associated with cytokine secretion disorders, which are associated with immune activation $(22,23)$. Drugs that can inhibit proinflammatory cytokines may produce antidepressant effects (24-26).
Furthermore, many studies have found that TRD is also accompanied by inflammatory dysregulation. Antidepressant-induced remission of depressive symptoms has also been associated with significant decreases in pro-inflammatory cytokine levels $(27,28)$. Major depressive patients with a history of non-response to antidepressants were found to have increased plasma concentration of IL-1, IL-6 and acute phase reactants compared with treatment-responsive patients $(29,30)$. Similarly, patients with increased inflammatory cytokines before treatment have been reported to be less responsive to antidepressant treatment $(31,32)$. Aspirin is a non-selective COX inhibitor with a broad spectrum of pharmacological 


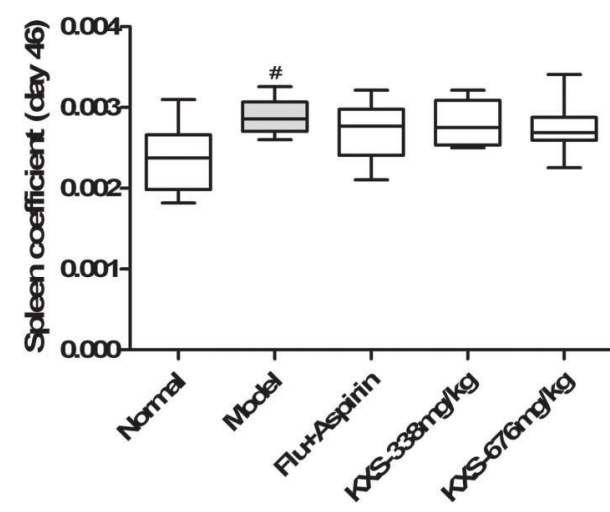

Figure 3. Effects of Kai-Xin-San (KXS) on spleen coefficient in fluoxetine-resistant depression (FRD) rats exposed to chronic and unpredictable mild stress model and randomly divided into four groups: FRD model control (Model), oral fluoxetine and aspirin (Flu + Aspirin), oral KXS at a dose of $338 \mathrm{mg} / \mathrm{kg}$, and $676 \mathrm{mg} / \mathrm{kg}$. Data are reported as means $\pm S D, n=12$. ${ }^{\#} P<0.05$ compared to untreated Normal group.

effects at multiple locations. Existing research shows that aspirin has antidepressant properties and accelerates antidepressant effects in preclinical models (33). Clinically, aspirin has been suggested to shorten the onset of action of selective reuptake inhibitors and to increase remission rates when added to fluoxetine in an open-label study of depressed patients previously non-responsive to fluoxetine alone (34). Therefore, it is essential to find effective treatments for TRD.

Traditional Chinese medicine in the treatment of depression is the focus of current research. It was

\section{References}

1. Trivedi MH, Rush AJ, Wisniewski SR, Nierenberg AA, Warden D, Ritz L, et al. Evaluation of outcomes with citalopram for depression using measurement-based care in $\mathrm{STAR}^{\star} \mathrm{D}$ : implications for clinical practice. Am J Psychiatry 2006; 163: 28-40, doi: 10.1176/appi.ajp.163.1.28.

2. Maes M, Bosmans E, De Jongh R, Kenis G, Vandoolaeghe E, Neels $H$. Increased serum IL-6and IL-1 receptor antagonist concentrations in major depression and treatment resistant depression. Cytokine 1997; 9: 853-858, doi: 10.1006/cyto. 1997.0238.

3. Sluzewska A, Sobieska M, Rybakowski JK. Changes in acute-phase proteins during lithium potentiation of antidepressants in refractory depression. Neuropsychobiol 1997; 35: 123-127, doi: 10.1159/000119332.

4. O'Brien SM, Scully P, Fitzgerald P, Scott LV, Dinan TG. Plasma cytokine profiles in depressed patients who fail to respond to selective serotonin reuptake inhibitor therapy. J Psychiatr Res 2007; 41: 326-331, doi: 10.1016/j.jpsychires. 2006.05.013.

5. Benedetti F, Lucca A, Brambilla F, Colombo C, Smeraldi E. Interleukine-6 serum levels correlate with response to documented that KXS could cure symptoms including desolation, moodiness, and forgetfulness, which are similar to symptoms of depression, such as depressed mood, anxiety, and impairment in learning and memory (13). As the principal herb of KXS, ginseng has been demonstrated to improve learning and memory in animals (10). 3,6'-Disinapoyl sucrose is an active oligosaccharide ester found in Polygala tenuifolia Willd, exhibits notable antidepressant effects in pharmacological depression models, and alleviates stress-induced behavioral abnormalities $(15,35)$.

CUMS model is accepted as a valuable method for inducing experimental depression in rats. At least 20-30\% of depressive rats do not respond to fluoxetine treatment (36). Aspirin can assist the treatment of depression through its anti-inflammatory effects, which led us to verify if KXS also played a role in regulating inflammatory pathways. Therefore, we studied the effect of KXS on cytokines in the hippocampus and serum of TRD rats.

The effects of KXS on the immune system of TRD rats are concentration-dependent and maximum inhibition of inflammatory factors and promotion of anti-inflammatory cytokines can be obtained by adjusting the dosage of KXS to optimize its antidepressant activity. These results suggest that administration of KXS for fluoxetine-resistant depression in rats was effective in improving depression by influencing the inflammatory processes.

\section{Acknowledgments}

This work was supported by grants from the National Natural Science Foundation (Nos. 81302909, 81373996, and 81573876). antidepressant sleep deprivation and sleep phase advance. Prog Neuropsychopharmacol Biol Psychiatry 2002; 26: 1167-1170, doi: 10.1016/S0278-5846(02)00255-5.

6. Lanquillon S, Krieg JC, Bening-Abu-Shach U, Vedder $\mathrm{H}$. Cytokine production and treatment response in major depressive disorder. Neuropsychopharmacol 2000; 22: 370-379, doi: 10.1016/S0893-133X(99)00134-7.

7. Mikova O, Yakimova R, Bosmans E, Kenis G, Maes M. Increased serum tumor necrosis factor alpha concentrations in major depression and multiple sclerosis. Eur Neuropsychopharmacol 2001; 11: 203-208, doi: 10.1016/S0924977X(01)00081-5.

8. Wang Y, Cui XL, Liu YF, Gao F, Wei D, Li XW, et al. LPS inhibits the effects of fluoxetine on depression-like behavior and hippocampal neurogenesis in rats. Prog Neuropsychophamacol Biol Psychiatry 2011; 35: 1831-1835, doi: 10.1016/ j.pnpbp.2011.07.004.

9. Dong $X Z$, Li ZL, Zheng $X L$, Mu LH, Zhang G, Liu P. A representative for emotional disease, Ding-Zhi-Xiao-Wan restores 5-HT system deficit through interfering the synthesis and transshipment in chronic mild stress-induced 
depressive rats. J Ethnopharmacol 2013; 150: 1053-1061, doi: 10.1016/j.jep.2013.10.018.

10. Zhou XJ, Liu M, Yan JJ, Can Y, Liu P. Antidepressant-like effect of the extracted of Kai Xin San, a traditional Chinese herbal prescription, is explained by modulation of the central monoaminergic neurotransmitter system in mouse. J Ethnophamacol 2012;139: 422-428, doi: 10.1016/j.jep. 2011.11.027.

11. Hu Y, Liu P, Guo DH, Rahman K, Wang DX, Chen ML, et al. Behavioral and biochemical effects of Kaixin-San, a traditional Chinese medicinal empirical formula. Drug Dev Res 2008; 69: 267-271, doi: 10.1002/ddr.20252.

12. Cao Y, Hu Y, Liu P, Zhao HX, Zhu XJ, Wei YM. Effects of a Chinese traditional formula Kai Xin San (KXS) on chronic fatigue syndrome mice induced by forced wheel running. J Ethnopharmacol 2012; 139: 19-25, doi: 10.1016/j.jep.2011. 08.030 .

13. Dang $H$, Sun $L$, Liu $X$, Peng $B$, Wang $Q$, Jia $W$, et al. Preventive action of Kai Xin San aqueous extract on depressive-like symptoms and cognition deficit induced by chronic mild stress. Exp Biol Med 2009; 234: 785-793, doi: 10.3181/0812-RM-354.

14. Mu LH, Huang ZX, Liu P, Hu Y, Gao Y. Acute and subchronic oral toxicity assessment of the herbal formula Kai-Xin-San. J Ethnopharmacol 2011; 138: 351-357, doi: 10.1016/j.jep. 2011.08.033.

15. Hu Y, Liu M, Liu P, Guo DH, Wei RB, Rahman K. Possible mechanism of the antidepressant effect of 3,6'-disinapoyl sucrose from Polygala tenuifolia Willd. J Pharm Pharmacol 2011; 63: 869-874, doi: 10.1111/j.2042-7158.2011.01281.x.

16. Chung YC, Kim SR, Park JY, Chung ES, Park KW, Won SY. Fluoxetine prevents MPTP-induced loss of dopaminergic neurons by inhibiting microglial activation. Neuropharmacol 2011; 60: 963-974, doi: 10.1016/j.neuropharm.2011.01.043.

17. Musselman D, Royster EB, Wang M, Long Q, Trimble LM, Mann TK, et al. The impact of escitalopram on IL-2-induced neuroendocrine, immune, and behavioral changes in patients with malignant melanoma: preliminary findings. Neuropsychopharmacol 2013; 38: 1921-1928, doi: 10.1038/ npp.2013.85.

18. Wang Y, Yang F, Liu YF, Gao F, Jiang W. Acetylsalicylic acid as an augmentation agent in fluoxetine treatment resistant depressive rats. Neurosci Lett 2011; 499: 74-79, doi: 10.1016/ j.neulet.2011.05.035.

19. Jadad AR, Moore RA, Carroll D, Jenkinson C, Reynolds DJM, Gavaghan DJ, et al. Assessing the quality of reports of randomized clinical trials: is blinding necessary? Control Clin Trials 1996; 17: 1-12, doi: 10.1016/0197-2456(95)00134-4.

20. Schulz KF, Chalmers I, Hayes RJ, Altman D. Empirical evidence of bias. Dimensions of methodological quality associated with estimates of treatment effects in controlled trials. JAMA 1995; 273: 408-412, doi: 10.1001/jama.1995. 03520290060030.

21. Einvik G, Vistnes M, Hrubos-Strom H, Randby A, Namtvedt SK, Nordhus IH, et al. Circulating cytokine concentrations are not associated with major depressive disorder in a community based cohort. Gen Hosp Psychiat 2012; 34: 262-267, doi: 10.1016/j.genhosppsych.2012.01.017.

22. Irwin MR, Miller AH. Depressive disorders and immunity: 20 years of progress and discovery. Brain Behav Immun 2007; 21: 374-383, doi: 10.1016/j.bbi.2007.01.010.
23. Krishnan V, Nestler EJ. The molecular neurobiology of depression. Nature 2008; 455: 894-902, doi: 10.1038/ nature 07455 .

24. Amini H, Aghayan S, Jalili SA, Akhondzadeh S, Yahyazadeh O, Pakravan-Nejad M. Comparison of mirtazapine and fluoxetine in the treatment of major depressive disorder: a double-blind, randomized trial. J Clin Pharm Ther 2005; 30: 133-138, doi: 10.1111/j.1365-2710.2004. 00585.x.

25. Wheatley DP, van Moffaert M, Timmerman L, Kremer CM. Mirtazapine: efficacy and tolerability in comparison with fluoxetine in patients with moderate to severe major depressive disorder. Mirtazapine-Fluoxetine Study Group. J Clin Psychiat 1998; 59: 306-312, doi: 10.4088/JCP. v59n0606.

26. Versiani M, Moreno R, Ramakers-van Moorsel CJA, Schutte AJ. Comparative Efficacy Antidepressants Study Group. Comparison of the effects of mirtazapine and fluoxetine in severely depressed patients. CNS Drugs 2005; 19: 137146, doi: 10.2165/00023210-200519020-00004.

27. Leo R. Di Lorenzo G, Tesauro M, Razzini C, Forleo GB, Chiricolo G, et al. Association between enhanced soluble CD40 ligand and proinflammatory and prothrombotic states in major depressive disorder: pilot observations on the effects of selective serotonin reuptake inhibitor therapy. J Clin Psychiatry 2006; 67: 1760-1766, doi: 10.4088/JCP. v67n1114.

28. Pizzi C, Mancini S, Angeloni L, Fontana F, Manzoli L, Costa GM. Effects of selective serotonin reuptake inhibitor therapy on endothelial function and inflammatory markers in patients with coronary heart disease. Clin PharmacolTher 2009; 86: 527-532.

29. Maes M, Bosmans E, De Jongh R, Kenis G, Vandoolaeghe $E$, Neels $H$. Increased serum IL-6 and IL- 1 receptor antagonist concentrations in major depression and treatment resistant depression. Cytokine 1997;9: 853-858, doi: 10.1006/cyto.1997.0238.

30. Sluzewska A, Sobieska M, Rybakowski JK. Changes in acute-phase proteins during lithium potentiation of antidepressants in refractory depression, Neuropsychobiology 1997; 35:123-127.

31. Benedetti F, Lucca A, Brambilla F, Colombo C, Smeraldi E. Interleukine-6 serum levels correlate with response to antidepressant sleep deprivation and sleep phase advance. Prog Neuropsychopharmacol Biol Psychiatry 2002; 26: 1167-1170, doi: 10.1016/S0278-5846(02)00255-5

32. Lanquillon S, Krieg JC, Bening-Abu-Shach $\mathrm{U}$, Vedder $\mathrm{H}$. Cytokine production and treatment response in major depressive disorder. Neuropsychopharmacology 2000; 22: 370-379, doi: 10.1016/S0893-133X(99)00134-7.

33. Brunello N, Alboni S, Capone G, Benatti C, Blom JM, Tascedda $F$, et al. Acetylsalicylic acid accelerates the antidepressant effect of fluoxetine in the chronic escape deficit model of depression. Int Clin Psychopharmacol 2006; 21: 219-225, doi: 10.1097/00004850-20060700000004.

34. Mendlewicz J, Kriwin P, Oswald P, Souery D, Alboni S, Brunello N. Shortened onset of action of antidepressants in major depression using acetylsalicylic acid augmentation: a pilot open-label study. Int Clin Psychopharmacol 2006; 21: 227-231, doi: 10.1097/00004850-200607000-00005. 
35. Hu Y, Liao HB, Guo DH, Liu P, Wang YY, Rahman K. Antidepressant-like effects of $3,6^{\prime}$-disinapoyl sucrose on hippocampal neuronal plasticity and neurotrophic signal pathway in chronically mild stressed rats. Neurochem Int 2010; 56: 461-465, doi: 10.1016/j.neuint.2009.12.004.
36. Bergstrom A, Jayatissa MN, Thykjaer T, Wiborg O. Molecular pathways associated with stress resilience and drug resistance in the chronic mild stress rat model of depression: a gene expression study. J Mol Neurosci 2007; 33: 201-215, doi: 10.1007/s12031-007-0065-9. 